\title{
Stage III Cervical Cancer FIGO 2018
}

National Cancer Institute

\section{Source}

National Cancer Institute. Stage III Cervical Cancer FIGO 2018. NCI Thesaurus. Code

C162239.

Cervical carcinoma that involves the lower third of the vagina and/or extends to the pelvic wall and/or causes hydronephrosis or nonfunctioning kidney and/or involves pelvic and/or para-aortic lymph nodes. (FIGO 2018) 